\title{
Body Image Disturbances and Weight Bias After Obesity Surgery: Semantic and Visual Evaluation in a Controlled Study, Findings from the BodyTalk Project
}

\author{
Paolo Meneguzzo ${ }^{1}$ (D) Simone Claire Behrens ${ }^{2,3}$ (D) $\cdot$ Angela Favaro ${ }^{1,4}\left(\right.$ D $\cdot$ Elena Tenconi ${ }^{1,4}\left(\right.$ D $\cdot$ Vincenzo Vindigni $^{1}$ (D) \\ Martin Teufel $^{5}$ (D) Eva-Maria Skoda ${ }^{5}$. Marion Lindner ${ }^{5} \cdot$ M. Alejandra Quiros-Ramirez ${ }^{3,6} \cdot$ Betty Mohler $^{3}$. \\ Michael Black ${ }^{3} \cdot$ Stephan Zipfel ${ }^{2}$ (D) $\cdot$ Katrin E. Giel $^{2} \cdot$ Chiara Pavan $^{7}$
}

Received: 13 July 2020 /Revised: 1 December 2020 / Accepted: 9 December 2020 / Published online: 6 January 2021

(C) The Author(s) 2021

\begin{abstract}
Purpose Body image has a significant impact on the outcome of obesity surgery. This study aims to perform a semantic evaluation of body shapes in obesity surgery patients and a group of controls.

Materials and Methods Thirty-four obesity surgery (OS) subjects, stable after weight loss (average $48.03 \pm 18.60 \mathrm{~kg}$ ), and 35 overweight/obese controls (MC), were enrolled in this study. Body dissatisfaction, self-esteem, and body perception were evaluated with self-reported tests, and semantic evaluation of body shapes was performed with three specific tasks constructed with realistic human body stimuli.

Results The OS showed a more positive body image compared to HC $(p<0.001)$, higher levels of depression $(p<0.019)$, and lower self-esteem $(p<0.000)$. OS patients and $\mathrm{HC}$ showed no difference in weight bias, but OS used a higher BMI than $\mathrm{HC}$ in the visualization of positive adjectives $(p=0.011)$. Both groups showed a mental underestimation of their body shapes.

Conclusion OS patients are more psychologically burdened and have more difficulties in judging their bodies than overweight/ obese peers. Their mental body representations seem not to be linked to their own BMI. Our findings provide helpful insight for the design of specific interventions in body image in obese and overweight people, as well as in OS.
\end{abstract}

Keywords Body image $\cdot$ Obesity $\cdot$ Obesity surgery $\cdot$ Weight bias $\cdot$ Body weight dissatisfaction

Paolo Meneguzzo

meneguzzo.p@gmail.com

1 Department of Neuroscience, University of Padova, via Giustiniani 2, 35128 Padova, Italy

2 Department of Psychosomatic Medicine and Psychotherapy, Medical University Hospital Tübingen, Tübingen, Germany

3 Max Planck Institute for Intelligent Systems, Tübingen, Germany

4 Padova Neuroscience Center, University of Padova, Padova, Italy

5 Clinic for Psychosomatic Medicine and Psychotherapy, University of Duisburg-Essen, LVR University-Hospital Essen, Essen, Germany

6 Psychology Department, University of Konstanz, Konstanz, Germany

7 Department of Medicine, University of Padova, Padova, Italy

\section{Background}

A significant proportion of patients with obesity surgery (20$30 \%$ ) experience an unfavorable outcome within the first postoperative years [1], and successful weight loss is not necessarily a predictor of improvement of the psychosocial outcome [2]. A potential mechanism could be body image, which not only drives approximately $30 \%$ of individuals' choices about food consumption [2-4] but also includes cognitions and attitudes about the self and how it relates to social standards. In this point of view, patients seeking contouring surgery represent an interesting population due to their exposure to body changes and the needing of specific interventions on body image $[5,6]$.

Body image has been defined as the multidimensional construct derived from the perceptions, thoughts, and feelings associated with the body and bodily experiences [7, 8]. Body image is not only the satisfaction/ dissatisfaction with one's body; it also has a cognitive component that reflects the relationship between the 
evaluation of body shapes and the positive/negative valence attributed to them [9]. Body judgments are characterized by increasing rates of prejudice and discrimination toward overweight people, even if there is an increasing rate of overweight in the population [10]. Mental health is compromised by negative body image aspects, with an increase of psychopathological symptomatology, from depression to eating dysfunctional behaviors $[11,12]$.

Obese and overweight people are more exposed to negative evaluations of their body and their selves, with dramatic impacts on their everyday lives, especially in their perceptions of sizes, spaces, and attitudes [13-15]. Moreover, even though obese patients often experience an improvement of their body images after obesity surgery [16], patients could suffer the consequences of the internalization of weight stigmatization with weight gain or treatment drop out [17-20]. Indeed, obese and overweight patients exhibit specific dysfunctional cognitive body schemata that are not easily detachable from body image construct, and that are different from the normal weight population ones [21]. An implicit or explicit weight stigmatization could bring people to feel more "disgust," "blame," or "contempt" for overweight bodies - both their own bodies and others-with an influence on behaviors and mental health [22-25]. Such effects could interfere with improving body image after obesity surgery, and, in a second step, reduce mental health and thereby compromise the weight loss [19].

Excess skin cannot be reduced with physical activities [26], and on top of the nursing challenges, it constantly reminds a patient to the fact that he or she has been extremely obese. Literature has shown that even after contouring surgery, patients showed a low satisfaction with their own body, with a significant role of expectation on the judgment of the results [27, 28]. However, it so far remains open how these patients actually perceive their body as compared to other bodies, and how their body image relates to the body image of people who lack experience of obesity surgery.

To meet this question, this study takes a comprehensive approach to investigate body image of obesity surgery patients looking for a contouring surgery. Using established psychometric instruments as well as computerized experimental approaches, different facets of body image construct have been evaluated, comparing overweight-obese people with and without a history of obesity surgery. Our hypothesis is that OS patients could show an impaired body image evaluation and cognitive representation, due to their history of significant weight fluctuations. Moreover, different semantic evaluation of body shapes could be found between included samples, which could be a possible target of intervention.

\section{Methods}

\section{Participants}

Thirty-four people ( 2 male, 32 female) with previous obesity surgery interventions for severe obesity seeking a contouring surgery were recruited in the outpatient service of the Plastic Surgery Unit of the University of Padova. Obesity surgery patients were recruited between 2 and 10 years after their laparoscopic sleeve gastrectomy; they all had stable weights for, at least, 6 months. A group of 35 BMI-matched controls (MC) of matched age and BMI were selected from a sample of international subjects who participated in a research project about body image evaluation ( 3 male, 32 female), called BodyTalk project [29]. The inclusion criteria for both groups were an age between 18 and 65 years and no severe mental and medical comorbidity (e.g., no eating disorders), no neurological trauma and disorders, nor drug addictions. The exclusion criteria for the MC were that they could not have a history of obesity surgery interventions or desire to have any. Informed consent was collected from each participant. The study was approved by the local ethic committee as part of a larger study on the cognitive evaluation of obesity surgery patients, and it complies with the provisions of the Declaration of Helsinki.

\section{Assessment Instruments}

OS participants were evaluated by a trained researcher with clinical interviews, assessing weight history, behaviors and previous psychological and surgery interventions, and exclusion and inclusion criteria were applied. After providing informed consent, included participants were administered specific questionnaires about eating and weight concerns, depression, self-esteem and body evaluation, and afterwards completed three computerized tasks. The MC were tested with the same tasks using the same methodology across the different centers. The following instruments were used.

The Rosenberg self-esteem scale (RSES) [30] is a wellestablished self-reported 10-item test about self-esteem. For each item, there is a Likert scale response from "strongly agree" to "strongly disagree." Results higher than 15 may indicate seriously low self-esteem.

The physical appearance comparison scale (PACS) [31] is a 5-item self-reported test used to assess the degree of physical comparison with others in various social situations. Responses are collected on a Likert scale, ranging from "never" to "always." Higher scores indicate higher social comparison tendencies.

The body dissatisfaction subscale and the drive to thinness subscale of the eating disorder inventory (EDI) [32] are both well-established measures of specific psychopathology constructs about body shapes. Each item is on a 6-point scale, ranging from "always" to "never" and rated $0-3$, and higher scores indicate higher body dissatisfaction. 
The patient health questionnaire (PHQ-9) [33] is a 9-item self-reported test, and it is considered a good measure of depression. There are four answer categories from "not at all" to "almost every day", and higher total scores indicate higher depression symptomatology.

The body image questionnaire (BIQ-20) [34] is a measure of the dynamism of one's body (e.g., "I feel very fit"-perception of body dynamics (PBD) subscale), as well as of its rejection (e.g., "My body often annoys me"-negative evaluation of the body (NEB) subscale). There are five answer categories, ranging from "Not true" to "Absolutely true," and higher scores indicate a more negative body image. The translation from the original Germany version to the Italian version was performed independently by an author and a professional translator, and the two versions were reviewed by two different German-speaking authors. A backward translation was performed in order to evaluate the semantic value of the questionnaire. Good reliability was found: PBD Cronbach's $\alpha=0.81$ and NEB Cronbach's $\alpha=0.85$.

\section{Study Design}

Semantic evaluation of body shapes and weight bias were assessed using a computerized evaluation of body shapes, as already applied to other clinical population [29]. The body shape evaluation was composed of three different tasks performed by participants and recorded using a 17" laptop in the morning. See Fig. 1 for a visual representation.

1. In the first task, the rating task, 12 body images, and 16 adjectives were presented to participants and asked to evaluate how the match was adequate. The image set was composed of a realistic human body stimulus of different weights: underweight, normal weight, overweight, and obese [35]. We selected the bodies to cover a range in BMI from 15.5 to $36.5 \mathrm{~kg} / \mathrm{m}^{2}$. The adjective set was composed of 16 adjectives describing both physical and behavioral factors selected from literature about weight bias and already used in other studies: active, apple-shaped, attractive, clumsy, determined, feminine, heavy-set, hourglass-shaped, impulsive, insecure, lazy, open-minded, pear-shaped, smart, thin, and unfriendly [36]. The adjectives were translated into Italian by a professional translator and an author independently and then checked by another author to evaluate the differences. A third author then translated back the adjectives in order to evaluate semantic correspondence. All possible combinations were presented randomly to the participants, and the evaluation was performed with a 4-point Likert scale from "very much" to "not at all."

2. In the second task, the adjustment task, an adapted version of the body shape visualization tool, was used [36]. The participants were asked to modify a digital, realistic neutral human presented on the computer screen using eight scrollbars, each representing a principal component of the body. The goal of this task was to generate a biometrically plausible 3D body model for each specific adjective from the adjective set. The first trial was carried out without providing any adjectives, and it was used to familiarize the participants with the scrollbars. Subsequently, each adjective from the first task set was presented randomly, and participants were asked to generate a prototypic body matching the adjective. At the end of the task, participants were asked to reproduce their own body.

3. In the third task, the valence assessment task, people were asked to evaluate all the presented adjective with a 5-point Likert scale from "clearly negative" to "clearly positive," as a measurement of adjectives valence. Adjectives were presented to all groups in a randomized order.

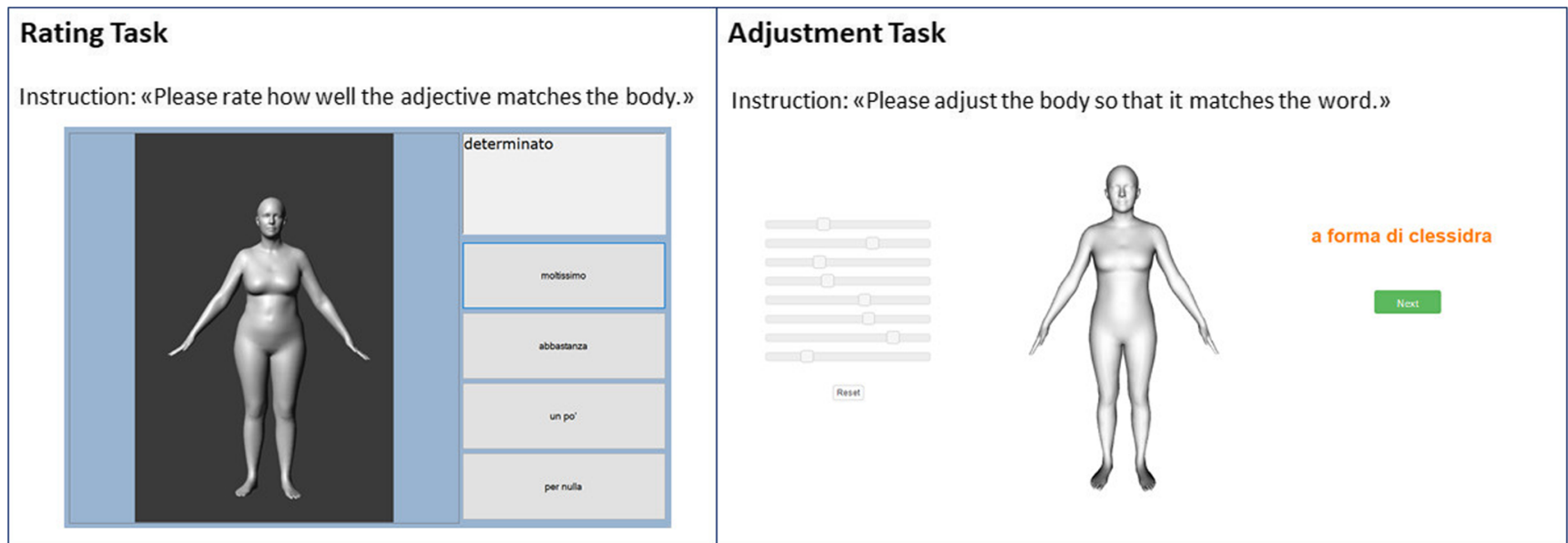

Fig. 1 Illustration of the two computerized tasks that were used for the assessment. The rating test consisted of 12 different bodies and 16 adjectives. The adjustment task was performed for the 16 adjectives and then for the visual representation of the patient's or control's own body. The order of the bodies/adjectives was randomized 


\section{Statistical Analysis}

The analysis was conducted with IBM SPSS Statistics 25.0 (SPSS, Chicago, IL, USA). A normal distribution test was performed for all variables. The demographic variables and questionnaire scores were tested using independent $t$-tests. To analyze semantic evaluation of body shape, we estimated the BMI of the generated bodies and compared them between the groups.

In the adjustment task, the height, in meters, of the human model was calculated subtracting its lowest point from its highest point. The volume in cubic meters of the models was calculated as described by Zhang and Chen [37], and, dividing its volume by the average human body density $\left(1010 \mathrm{~kg} / \mathrm{m}^{3}\right)$ [38] was obtained the weight of the models and was calculated the BMI.

In the valence assessment, no differences between subgroups emerged in the analysis of the assigned adjective valence (results from the valence assessment task). Adjectives were then grouped by the valences, from negative to positive according to participants' ratings, and the average BMIs were evaluated in order to look at the mental representation of body shapes.

To evaluate weight bias, data from the rating task were aggregated with data from the valence assessment task. Thus, we obtained the valence values for body weight categories (i.e., underweight, normal weight, overweight, and obese) and we were able to split them into positive/ negative adjectives. The normally distributed data was analyzed using $t$ tests. Pearson's correlation analysis was used to evaluate how the BMI of rated body shapes was associated with valence of the adjective. Comparisons between correlations were performed using the Fisher's $r$ to $\mathrm{z}$ transformation [39].
Body size estimation accuracy was assessed using the body perception index. It was calculated as the percentage of the ratio between the estimated BMI through the visualization tool and the actual BMI, in order to compare body perception [40]. Linear regression analysis were performed between actual BMI and own represented BMI, with the goal to evaluate if the accuracy could be explained by the participants' BMI.

Several regression analyses were also performed between the human model's BMI and psychological scores with stepwise approaches. The alpha was set at 0.05 for all the analyses. The effect sizes were calculated with Cohen's delta.

\section{Results}

A summary of the demographic characteristics and questionnaire scores of each group can be found in Table 1. Levels of self-esteem, body dissatisfaction, drive for thinness, depression, and body perception were found to be significantly different in the two investigated groups. The OS patients and MC had similar BMIs, but OS had a significantly higher lifetime maximum weight than $\mathrm{MC}(p<0.001)$. The OS patients reported a less negative body dissatisfaction than MC with EDI subscales $(p=0.001)$, but no difference was recorded about negative body evaluation using the BIQ-20 scale between the two groups, even if OS patients perceived their body as less dynamic $(p=0.002)$. Moreover, OS patients showed lower self-esteem $(p<0.001)$ and more depressive symptoms $(p=$ $0.019)$ than control peers.

The analysis of the semantic evaluation of body shape (adjustment task) suggested the presence of very similar specific body shape images linked to specific adjectives in both two
Table 1 Demographic characteristics of the included samples

\begin{tabular}{lllrrr}
\hline & OS (SD) $n=34$ & MC (SD) $N=35$ & $t$ & \multicolumn{1}{l}{$p$} & $d$ \\
\hline Age, years & $48.48(12.60)$ & $42.43(14.50)$ & 1.833 & 0.071 & 0.445 \\
Women, \% & 96.88 & 94.44 & & & \\
BMI, kg/m ${ }^{2}$ & $29.33(5.02)$ & $32.83(13.52)$ & -1.396 & 0.160 & 0.343 \\
BMI max lifetime, kg/m ${ }^{2}$ & $46.43(8.81)$ & $34.56(14.09)$ & -4.212 & $<0.001$ & 1.010 \\
BMI min after puberty, $\mathrm{kg} / \mathrm{m}^{2}$ & $24.62(5.65)$ & $22.24(4.24)$ & -1.979 & 0.052 & 0.476 \\
RSES & $17.21(2.06)$ & $21.26(4.54)$ & -4.780 & $<0.001$ & 1.148 \\
PACS & $13.73(4.66)$ & $14.66(3.15)$ & -0.969 & 0.336 & 0.234 \\
EDI-2 Drive for thinness & $7.56(5.36)$ & $14.32(12.70)$ & -2.896 & 0.006 & 0.693 \\
EDI-2 Body dissatisfaction & $10.27(6.56)$ & $22.71(18.83)$ & -3.678 & 0.001 & 0.882 \\
PHQ9 & $7.06(4.13)$ & $5.06(2.61)$ & 2.405 & 0.019 & 0.579 \\
BIQ-PBD & $30.52(4.93)$ & $35.04(6.48)$ & -3.229 & 0.002 & 0.785 \\
BIQ-NEB & $29.70(3.90)$ & $27.89(10.46)$ & 0.935 & 0.353 & 0.229 \\
\hline
\end{tabular}

$S D$ standard deviation, $O S$ obesity surgery subjects, $M C$ BMI-matched control, BMI body mass index, RSES Rosenberg Self-Esteem Scale, PACS Physical Appearance Comparison Scale, EDE- $Q$ Eating Disorder Examination Questionnaire, $P H Q$ Patient Health Questionnaire, $B I Q$ body image questionnaire, $P B D$ perception of body dynamics subscale, $N E B$ negative evaluation of the body subscale 
groups. The 3D body shape models' BMI are reported in Table 2. Only for specific adjectives (i.e., heavy-set and thin), the analysis have reported different BMI between OS patients and MC. Looking at specific adjectives, the analysis of the modification of the body components showed that between OS and controls different body parts are differently adjusted, even if the final BMIs are the same, see Fig. 2 for a visual representation of adjectives. For example, it is possible to evaluate that comparing OS and controls, the body model for "thin" was quite globally modified differently by OS that adjusted a fuller body, and, instead, for "lazy" the OS patients create a body model with a similar but with different hips and abdomen. Moreover, looking at the "own body" models, the OS patients showed a greater focused about their waists than the controls.

The assessment of weight bias through a correlation analysis between the BMI of the generated body shapes and adjective rating are reported in Table 3. This analysis suggests that participants were more willing to accept positive adjectives as a match when the BMI was low, and assigned negative adjectives as a match when the BMI was high. Both groups showed the same trends for a weight bias, even though the correlation factors exhibited different associations between BMI and valences. Comparisons between correlation coefficients (Fisher's $\mathrm{r}$ to $\mathrm{z}$ transformation) showed no significant differences (negative adjectives: $Z=0.244$, $p=0.807$; neutral: $Z=-1.068, p=0.286$; positive: $Z=-$ $0.262, p=0.793$ ).

The results from the modified own body shape were grouped according to the adjective valences (see Table 4).
This grouping revealed how the represented BMIs exhibited differences between the OS and MC participants only with positive adjectives. Moreover, the OS participants modified the human body model building higher BMI.

Moreover, the own body assessments of the OS patients were significantly different for the real BMI and reconstructed $\operatorname{BMI}(t(33)=3.414, p=0.002, d=0.786)$, but for the MC, the difference between the assessments of the two BMI categories was only weakly significant $(t(33)=2.072, p=0.050$, $d=0.368$ ). Body perception indices in OS patients results $89.62( \pm 18.24)$, and in MC it was $86.28( \pm 21.18)$, with a non-significant difference between them $(t(62)=$ $-0.679, p=0.500$ ).

Regression analysis showed that between actual BMI and own represented BMI, there is no relationship in OS patients $(F(1,32)=0.549, p=0.464)$, but it is present in MC $\left(F(1,33)=26.692, p<0.001, R^{2}=0.447\right)$. While in OS patients, actual BMI was not associated with represented BMI, MC tended to adjust the represented own body to higher BMI the higher their actual BMI was. See Fig. 3 for a graphical representation. Looking at relationship between represented own BMI and psychological features, no significant relationship has been found neither for OS nor for MC.

\section{Discussion}

Body image and shape evaluation is an important aspect in the psychopathology of severely obese patients and OS patients because it is strongly linked to intervention outcomes and
Table 2 BMI of realistic modified human bodies in the adjustment task

\begin{tabular}{lllrrl}
\hline & OS mean (SD) & MC mean (SD) & \multicolumn{1}{l}{ t } & \multicolumn{1}{l}{ p } \\
\hline Active & $22.39(3.10)$ & $21.28(2.77)$ & 1.478 & 0.144 & 0.378 \\
Apple shaped & $32.07(5.27)$ & $33.28(5.99)$ & -0.851 & 0.398 & 0.214 \\
Attractive & $22.11(2.88)$ & $22.30(2.83)$ & -0.260 & 0.796 & 0.067 \\
Clumsy & $30.44(5.18)$ & $29.11(6.04)$ & 0.940 & 0.351 & 0.281 \\
Determined & $23.86(3.30)$ & $22.18(2.73)$ & 2.168 & 0.034 & 0.555 \\
Feminine & $21.81(3.35)$ & $22.20(2.94)$ & -0.476 & 0.636 & 0.124 \\
Heavy set & $30.25(4.10)$ & $34.89(5.90)$ & -3.678 & 0.000 & 0.913 \\
Hourglass shaped & $24.50(4.32)$ & $23.62(5.21)$ & 0.733 & 0.466 & 0.184 \\
Impulsive & $24.49(3.14)$ & $26.11(4.63)$ & -1.589 & 0.119 & 0.410 \\
Insecure & $29.75(5.09)$ & $29.14(5.76)$ & 0.446 & 0.657 & 0.112 \\
Lazy & $30.71(5.03)$ & $31.54(6.66)$ & -0.550 & 0.585 & 0.141 \\
Open minded & $25.88(3.22)$ & $24.18(2.21)$ & 2.371 & 0.021 & 0.616 \\
Pear shaped & $26.20(3.49)$ & $25.68(3.91)$ & 0.555 & 0.581 & 0.140 \\
Smart & $24.49(2.00)$ & $24.36(2.00)$ & 1.207 & 0.233 & 0.065 \\
Thin & $19.63(3.24)$ & $17.10(1.92)$ & 3.852 & 0.000 & 0.950 \\
Unfriendly & $24.01(4.15)$ & $22.93(6.48)$ & 0.769 & 0.446 & 0.198 \\
Own body & $25.95(4.37)$ & $29.41(6.98)$ & -2.294 & 0.027 & 0.594 \\
\hline
\end{tabular}

OS obesity surgery subject, $M C$ BMI-matched control, $B M I$ body mass index, $S D$ standard deviation 
Fig. 2 Visual representation of the adjustment task. The height and the BMI are reported for each figure. Colors indicate the difference between control's body model and OS' one: dark blue represents no differences, lighter colors showed more difference

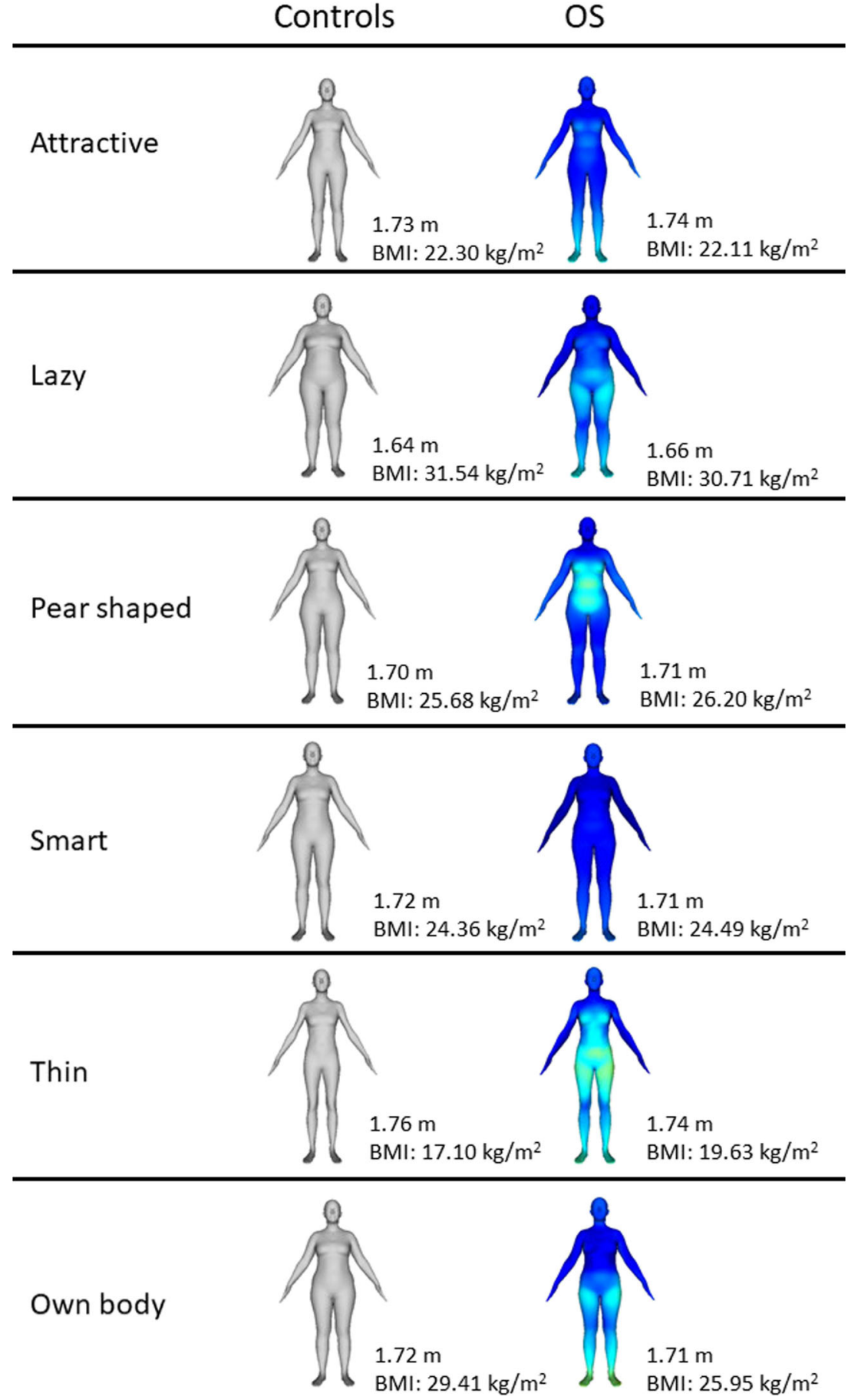

quality of life [27, 41]. To gain more insights on this important topic, we conducted a semantic evaluation of body shapes with a group of OS patients looking for contouring surgery who were compared to a group of weight and age-paired MC. It has already been demonstrated that obesity surgery intervention has a positive effect on body image [19, 42], but less is known about how people describe and evaluate body shapes and weights. Overall, our results confirmed that OS patients have a more positive image of their own body, corroborating data from OS literature [42], but we also showed specific aspects of the relationship between mental body representation and real body sizes.

The OS patients had lower self-esteem and higher levels of depression than the MC. Both factors are not clinically relevant according to standard scoring $[43,44]$, but they confirmed that OS patients should be considered vulnerable to psychological distress [45]. The drive for thinness and body dissatisfaction are bodily psychopathologies, and our results 
Table 3 Correlations analysis between rating task valences and BMI of the

\begin{tabular}{lll}
\hline & Adjective rating & Avatar BMI \\
\hline OS & Negative & $.337 * *$ \\
& Neutral & $.087 * *$ \\
& Positive & $-.295^{* *}$ \\
MC & Negative & $.281^{* *}$ \\
& Neutral & $.344^{* *}$ \\
& Positive & $-.233^{* *}$ \\
\hline
\end{tabular}

OS obesity surgery subject, $M C$ BMImatched control, $B M I$ body mass index; $* * p<.001$

are in line with data from literature: the OS participants had lower levels of bodily psychopathology and had the greatest weight loss [46]. Thus, even if the two groups have the same average BMI, losing weight could still reduce bodily focused psychopathology. However, OS still perceived lower body dynamics than their BMI peers, which is a construct that indicates how healthy, energetic, and confident a person feels about their body. This could be linked to the presence of excess skin that can modify the perception of one's own body movements, and justify the perceived need for a contouring surgery. This is in line with previous literature data that have shown that, even after obesity surgery, body image and the perception of body dynamic still remains poor comparable with peers without obesity surgery [16]. It could be the result of the previous morbid obesity, as well as a new problem linked to the body change [47]. Indeed, our sample was selected in the waiting list for contouring intervention for surplus skin and presented a maximum BMI — and then body sizes - significantly higher than MC with a possible impairment of every day movements. This aspect should be evaluated in future studies, with longitudinal design.

The results from the body evaluation showed that OS patients and MC do not show different relationships between negative or positive judgments and the mental shape representations, even though OS patients associate positive adjectives with a higher BMI than that produced in the models by MC. Also, the body area that OS patients changed in the human models showed a qualitative difference with the controls, showing a focusing on their waists. Body valence and judgment is a neglected topic in the OS literature, but the data showed that, after obesity surgery and weight loss, patients desire more unrealistic body shapes and this was interpreted as a higher adherence to the western thinideal $[19,48]$. The literature has already revealed that obesity surgery can positively change body image and body dissatisfaction [19, 49], our results added that these improvements could not be explained only by individual judgmental style about own body: severe obesity and the consequent obesity surgery seems to make the difference between people with the same BMI but with different weight and surgery history.

Our results are also in line with the literature that has demonstrated how overweight and obese people misperceived their weight, and that this aspect persists even after obesity surgery $[48,50,51]$. We do not find any difference in the underestimation of the own body sizes between subgroups, in accordance with the possible presence of a visual recalibration of the own

Table 4 Realistic human BMI and attributed valences, an integration of the adjustment, and valence tasks results

\begin{tabular}{|c|c|c|c|c|c|c|c|}
\hline & Valence (SD) & & OS (SD) & $\mathrm{MC}(\mathrm{SD})$ & $t$ & $p$ & $d$ \\
\hline Unfriendly & $-1.56(0.60)$ & Clearly negative & $24.01(4.15)$ & $22.93(6.48)$ & 0.769 & 0.446 & 0.198 \\
\hline $\begin{array}{l}\text { Insecure } \\
\text { Lazy }\end{array}$ & $\begin{array}{l}-1.07(0.61) \\
-1.02(0.66)\end{array}$ & Rather negative & $30.74(5.16)$ & $30.77(6.29)$ & -0.035 & 0.972 & 0.006 \\
\hline Clumsy & $-0.85(0.66)$ & & & & & & \\
\hline Apple shaped & $-0.63(0.76)$ & & & & & & \\
\hline $\begin{array}{l}\text { Heavy set } \\
\text { Pear shaped }\end{array}$ & $\begin{array}{l}-0.33(0.89) \\
-0.31(0.82)\end{array}$ & Neutral & $26.36(4.32)$ & $27.58(6.55)$ & -1.683 & 0.094 & 0.219 \\
\hline Impulsive & $-0.19(0.80)$ & & & & & & \\
\hline Hourglass shaped & $0.02(1.02)$ & & & & & & \\
\hline $\begin{array}{l}\text { Thin } \\
\text { Active }\end{array}$ & $\begin{array}{l}0.65(0.87) \\
1.33(0.61)\end{array}$ & Rather positive & $21.96(3.43)$ & $21.01(3.30)$ & 2.485 & 0.013 & 0.282 \\
\hline Attractive & $1.37(0.68)$ & & & & & & \\
\hline Feminine & $1.41(0.63)$ & & & & & & \\
\hline Determined & $1.43(0.60)$ & & & & & & \\
\hline Open minded & $1.52(0.69)$ & Clearly positive & $25.19(2.75)$ & $23.97(2.46)$ & 2.591 & 0.011 & 0.468 \\
\hline
\end{tabular}

$O S$ obesity surgery subject, $M C$ BMI-matched control, $S D$ standard deviation

In this table, adjectives has been aggregated based on the average valences attributed and the average adjustment BMI of the virtual body model has been calculated for each subgroups 


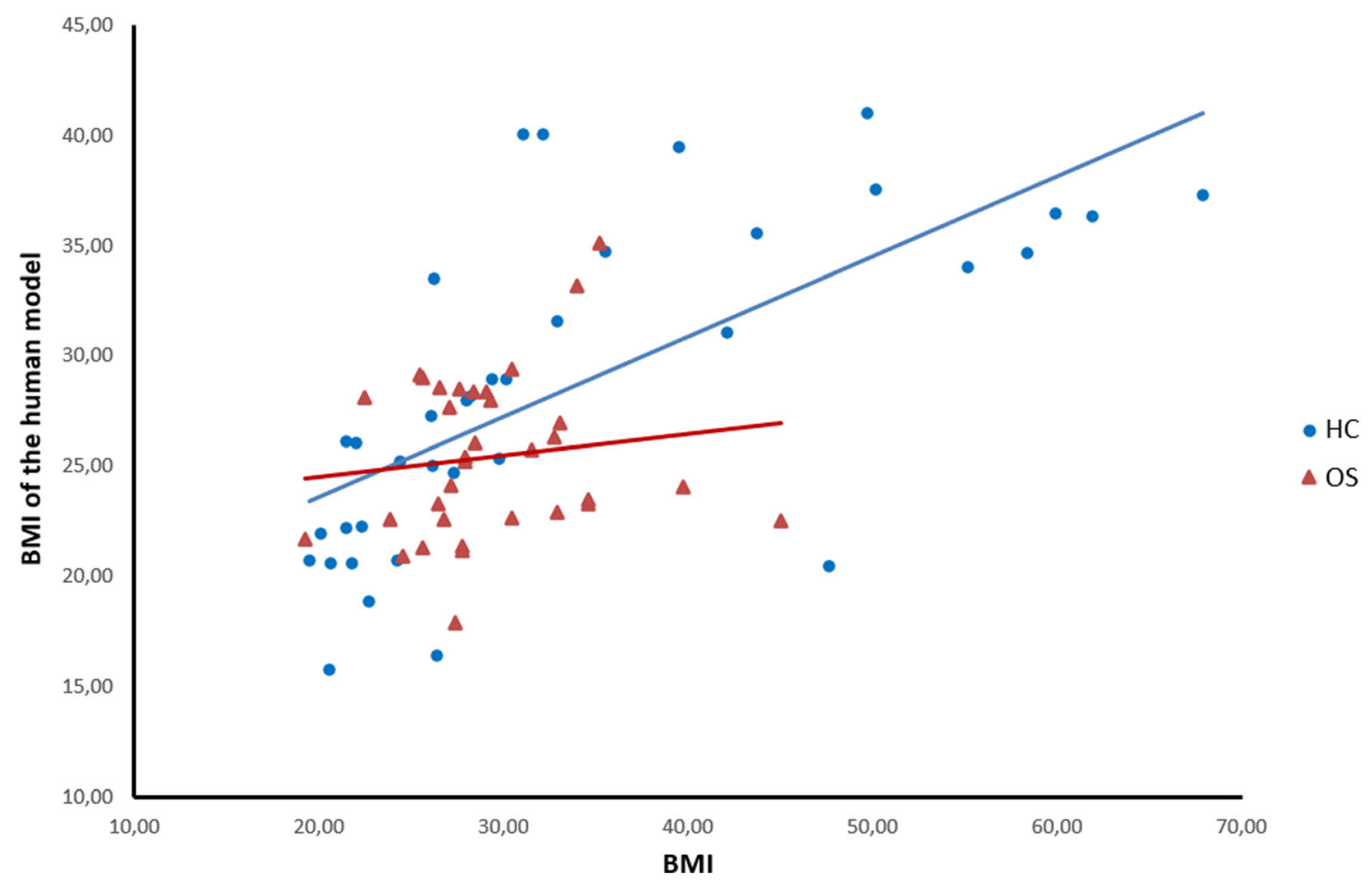

Fig. 3 The relationship between the actual BMI and the "own body" human model BMI by the adjustment task. The graphs show that a relationship between these two variables is maintained only in MC, and this could be interpreted as an impaired ability for OS to update their own body image

weight status in overweight people, called as "the visual normalization theory" $[52,53]$. However, in our sample, OS patients also showed a disruption of the relationship between the represented BMI and the actual BMI, which is preserved in MC, instead. Even with mirror evaluation, the literature has showed that ex-obese patients show a poorer estimation of their shapes compared to peers with the same BMI [54], as well as the impaired ability to notice weight gain in realistic 3D human models which represent themselves [55]. This impaired ability to update body image memory in OS patients could be explained by a sort of weight category bias, with OS patients that stopped to identify themselves as obese, a situation that they could linked to psychological burden situations, worsened by interpersonal problems, chronic stress, or anxiety [56]. This could have a significant implication for public health, because OS patients seem to be incapable of identifying weight changes after massive weight loss, as well as they seem to be into a weight category bias with a cognitive dissonance that could have a role in the weight reduction outcome, if it does not fit their goals [53].

However, the present findings should be viewed with caution. Our study is an observational one with a cross-sectional design, and thus, no inferences are possible about changes linked to the obesity surgery intervention. Results should be considered as preliminary due to the small sample included, that do not allow to perform a comparison between male/female. However, the selection of the MC paired to patients by age and weight should be considered a strength. Future research should be designed with a longitudinal approach with a larger sample of participants, covering the periods before and after obesity surgery and any counter-interventions.

In conclusion, our data suggest that body size representation and own body shape cognitive perception is complex, and maybe it is also a highly individual ability. Our data confirmed the impaired ability of obese and overweight patients to match actual body sizes with represented human body models, as well as showing a disrupted relationship between actual body and its mental representation in obesity surgery patients seeking for contouring surgery. Indeed, body image is an important factor for obesity surgery outcomes, especially in patients who are experiencing body changes and problematic excess skin. These results advocates for more studies about body image and body shape evaluation for people who undergo obesity surgery, as well as in overweight subjects seeking for weight interventions, because more data are needed.

Acknowledgments The authors would like to thank David Dal Brun for the translation of the tasks and questionnaires from German into Italian, and for the supervision in their linguistic adaptation.

Author Contributions PM: conceptualization; data curation; formal analysis; investigation; roles/writing - original draft. SCB: conceptualization; data curation; formal analysis; funding acquisition; investigation; methodology; roles/writing — original draft. AF: funding acquisition; supervision; validation; writing — review and editing. ET: resources; software; conceptualization; writing - review and editing. VV: funding acquisition; investigation; methodology. MT: funding acquisition; supervision; 
validation; writing - review and editing. EMS: data curation; writingreview and editing. ML: data curation; writing - review and editing. MAQR: conceptualization; software; supervision; visualization; writing - review and editing. BM: conceptualization; funding acquisition; supervision; writing - review and editing. MB: conceptualization; supervision; writing - review and editing. SZ: conceptualization; funding acquisition. KEG: conceptualization; funding acquisition; supervision; writing - review and editing. CP: conceptualization; funding acquisition; supervision; writing — review and editing.

\section{Compliance with Ethical Standards}

Conflict of Interest PM, SCB, AF, ET, VV, MT, EMS, ML, SZ, KEG, $\mathrm{CP}$ declare that they have no conflict of interest. MAQR has a patent WO2017129827A1 pending. BM reports grants from Volkswagen Foundation "Finding Perspective," outside the submitted work and is currently employed by Amazon Research Germany, but all of her commitments to this research were done prior to her employment. MB reports personal fees from Amazon, other from Meshcapade, outside the submitted work and has a patent "SKINNED MULTI-PERSON LINEAR MODEL" issued to Amazon and Meshcapade, a patent "Crowdshaping Realistic 3D Avatars with Words" pending but licensing status is unknown, and a various software and data licensed to Amazon and Meshcapade; moreover, reports corporate research funding: Intel, NVIDIA, Adobe, Facebook, and Amazon; financial interests: Amazon, Meshcapade; side employment: Amazon (20\%, current), Body Labs Inc. (20\%, 2013-2017); corporate boards: Body Labs Inc. (2013-2017).

Ethical Approval All procedures performed in studies involving human participants were in accordance with the ethical standards of the institutional and national research committee and with the 1964 Helsinki declaration and its later amendments or comparable ethical standards.

Informed Consent Informed consent was obtained from all individual participants included in the study.

Open Access This article is licensed under a Creative Commons Attribution 4.0 International License, which permits use, sharing, adaptation, distribution and reproduction in any medium or format, as long as you give appropriate credit to the original author(s) and the source, provide a link to the Creative Commons licence, and indicate if changes were made. The images or other third party material in this article are included in the article's Creative Commons licence, unless indicated otherwise in a credit line to the material. If material is not included in the article's Creative Commons licence and your intended use is not permitted by statutory regulation or exceeds the permitted use, you will need to obtain permission directly from the copyright holder. To view a copy of this licence, visit http://creativecommons.org/licenses/by/4.0/.

\section{References}

1. Sarwer DB, Allison KC, Wadden TA, et al. Psychopathology, disordered eating, and impulsivity as predictors of outcomes of bariatric surgery. Surg Obes Relat Dis. 2019;15:650-5. https://doi.org/ 10.1016/j.soard.2019.01.029.

2. Geller S, Dahan S, Levy S, et al. Body image and emotional eating as predictors of psychological distress following bariatric surgery. Obes Surg. 2019;30:1417-23. https://doi.org/10.1007/s11695-01904309-1.

3. Libeton M, Dixon JB, Laurie C, et al. Patient motivation for bariatric surgery: characteristics and impact on outcomes. Obes Surg. 2004;14:392-8. https://doi.org/10.1381/096089204322917936.
4. Friedman KE, Reichmann SK, Costanzo PR, et al. Body image partially mediates the relationship between obesity and psychological distress. Obes Res. 2002;10:33-41. https://doi.org/10.1038/ oby.2002.5.

5. Ghai A, Milosevic I, Laliberte M, et al. Body image concerns in obese women seeking bariatric surgery. Ethn Inequalities Heal Soc Care. 2014;7:96-107. https://doi.org/10.1108/EIHSC-11-20130044.

6. Sarwer DB, Polonsky HM. Body image and body contouring procedures. Aesthet Surg J. 2016;36:1039-47. https://doi.org/10.1093/ asj/sjw127.

7. Cash TF. Body image: past, present, and future. Body Image. 2004;1:1-5. https://doi.org/10.1016/S1740-1445(03)00011-1.

8. Altabe M, Thompson JK. Body image: a cognitive self-schema construct? Cognit Ther Res. 1996;20:171-93. https://doi.org/10. 1007/BF02228033.

9. Kling J, Kwakkenbos L, Diedrichs PC, et al. Systematic review of body image measures. Body Image. 2019;30:170-211. https://doi. org/10.1016/j.bodyim.2019.06.006.

10. O'Brien KS, Latner JD, Ebneter D, et al. Obesity discrimination: the role of physical appearance, personal ideology, and anti-fat prejudice. Int J Obes. 2013;37:455-60. https://doi.org/10.1038/ijo. 2012.52.

11. Shentow-Bewsh R, Keating L, Mills JS. Effects of anti-obesity messages on women's body image and eating behaviour. Eat Behav. 2016;20:48-56. https://doi.org/10.1016/j.eatbeh.2015.11. 012.

12. Ramos P, Moreno-Maldonado C, Moreno C, et al. The role of body image in internalizing mental health problems in spanish adolescents: an analysis according to sex, age, and socioeconomic status. Front Psychol. 2019;10 https://doi.org/10.3389/fpsyg.2019.01952.

13. O'Brien KS, Latner JD, Puhl RM, et al. The relationship between weight stigma and eating behavior is explained by weight bias internalization and psychological distress. Appetite. 2016;102:70 6. https://doi.org/10.1016/j.appet.2016.02.032.

14. Varns JA, Fish AF, Eagon JC. Conceptualization of body image in the bariatric surgery patient. Appl Nurs Res. 2018;41:52-8. https:// doi.org/10.1016/j.apnr.2018.03.008.

15. Puhl R, Luedicke J. Weight-based victimization among adolescents in the school setting: emotional reactions and coping behaviors. $\mathrm{J}$ Youth Adolesc. 2012;41:27-40. https://doi.org/10.1007/s10964011-9713-Z.

16. Teufel M, Rieber N, Meile T, et al. Body image after sleeve gastrectomy: reduced dissatisfaction and increased dynamics. Obes Surg. 2012;22:1232-7. https://doi.org/10.1007/s11695-012-06904.

17. Adami GF, Meneghelli A, Bressani A, et al. Body image in obese patients before and after stable weight reduction following bariatric surgery. J Psychosom Res. 1999;46:275-81. https://doi.org/10. 1016/S0022-3999(98)00094-4.

18. Griffiths $\mathrm{C}$, Williamson $\mathrm{H}$, Zucchelli $\mathrm{F}$, et al. A systematic review of the effectiveness of acceptance and commitment therapy (ACT) for body image dissatisfaction and weight self-stigma in adults. $\mathrm{J}$ Contemp Psychother. 2018;48:189-204. https://doi.org/10.1007/ s10879-018-9384-0.

19. Ivezaj V, Grilo CM. The complexity of body image following bariatric surgery: a systematic review of the literature. Obes Rev. 2018;19:1116-40. https://doi.org/10.1111/obr.12685.

20. Raves DM, Brewis A, Trainer S, et al. Bariatric surgery patients' perceptions of weight-related stigma in healthcare settings impair post-surgery dietary adherence. Front Psychol. 2016;7:1-13. https://doi.org/10.3389/fpsyg.2016.01497.

21. Irvine KR, McCarty K, McKenzie KJ, et al. Distorted body image influences body schema in individuals with negative bodily attitudes. Neuropsychologia. 2019;122:38-50. https://doi.org/10. 1016/j.neuropsychologia.2018.11.015. 
22. Vartanian LR, Thomas MA, Vanman EJ. Disgust, contempt, and anger and the stereotypes of obese people. Eat Weight Disord. 2013;18:377-82. https://doi.org/10.1007/s40519-013-0067-2.

23. Vartanian LR, Trewartha T, Vanman EJ. Disgust predicts prejudice and discrimination toward individuals with obesity. J Appl Soc Psychol. 2016;46:369-75. https://doi.org/10.1111/jasp.12370.

24. Wirtz C, van der Pligt J, Doosje B. Derogating obese individuals: the role of blame, contempt, and disgust. J Appl Soc Psychol. 2016;46:216-28. https://doi.org/10.1111/jasp.12357.

25. Gudzune KA, Bennett WL, Cooper LA, et al. Perceived judgment about weight can negatively influence weight loss: a cross-sectional study of overweight and obese patients. Prev Med (Baltim). 2014;62:103-7. https://doi.org/10.1016/j.ypmed.2014.02.001.

26. Colwell AS. Current concepts in post-bariatric body contouring. Obes Surg. 2010;20:1178-82. https://doi.org/10.1007/s11695010-0120-4.

27. de Zwaan M, Georgiadou E, Stroh CE, et al. Body image and quality of life in patients with and without body contouring surgery following bariatric surgery: a comparison of pre- and post-surgery groups. Front Psychol. 2014;5:1-10. https://doi.org/10.3389/fpsyg. 2014.01310.

28. Pecori L, Cervetti GGS, Marinari GM, et al. Attitudes of morbidly obese patients to weight loss and body image following bariatric surgery and body contouring. Obes Surg. 2007;17:68-73. https:// doi.org/10.1007/s11695-007-9008-3.

29. Behrens SC, Meneguzzo P, Favaro A, et al. Weight bias and linguistic body representation in anorexia nervosa: findings from the BodyTalk project. Eur Eat Disord Rev. 2020:1-12. https://doi.org/ 10.1002/erv.2812.

30. Rosenberg M. Society and the adolescent self-image; 1965 . https:// doi.org/10.1007/s12671-015-0407-6.

31. Thompson JK, Heinberg L, Tantleff-Dunn S. The Physical Appearance Comparison Scale (1991). Psychology Faculty Publications. 2116. Psychol Fac Publ 1991:2116.

32. Garner DM. EDI-2: Eating disorder inventory-2. Odessa: Psychological Assessment Resources, 1991.

33. Kroenke K, Spitzer RL. The PHQ-9: a new depression diagnostic and severity measure. Psychiatr Ann. 2002;32:509-15. https://doi. org/10.3928/0048-5713-20020901-06.

34. Clement, Ulrich, and Bernd Löwe. Die Validierung des FKB-20 als Instrument zur Erfassung von Körperbildstörungen bei psychosomatischen Patienten. PPmP: Psychotherapie Psychosomatik Medizinische Psychologie 1996.

35. Loper M, Mahmood N, Romero J, et al. SMPL: A skinned multiperson linear model. ACM Trans Graph. 2015;34:1-16. https://doi. org/10.1145/2816795.2818013.

36. Streuber S, Quiros-Ramirez MA, Hill MQ, et al. Body talk: Crowdshaping realistic 3D avatars with words. ACM Trans Graph. 2016;35:1-14. https://doi.org/10.1145/2897824.2925981.

37. Zhang $\mathrm{C}$, Chen T. Efficient feature extraction for $2 \mathrm{D} / 3 \mathrm{D}$ objects in mesh representation. IEEE Int Conf Image Process. 2001;3:935-8. https://doi.org/10.1109/icip.2001.958278.

38. Satoh H. Collection of ergonomic data and equations. Tokyo: Gihoudou; 1992.

39. Cohen P. Applied multiple regression/correlation analysis for the behavioral sciences: Routledge; 2014. https://doi.org/10.4324/ 9781410606266.

40. Slade PD, Russell GFM. Awareness of body dimensions in anorexia nervosa: cross-sectional and longitudinal studies. Psychol Med. 1973:3:188-99. https://doi.org/10.1017/S0033291700048510.
41. Song AY, Rubin JP, Thomas V, et al. Body image and quality of life in post massive weight loss body contouring patients. Obesity. 2006;14:1626-36. https://doi.org/10.1038/oby.2006.187.

42. Sarwer DB, Steffen KJ. Quality of life, body image and sexual functioning in bariatric surgery patients. Eur Eat Disord Rev. 2015;23:504-8. https://doi.org/10.1002/erv.2412.

43. Manea L, Gilbody S, McMillan D. Optimal cut-off score for diagnosing depression with the patient health questionnaire (PHQ-9): a meta-analysis. Can Med Assoc J. 2012;184:281-2. https://doi.org/ 10.1503/cmaj.112004.

44. Klaczynski PA, Goold KW, Mudry JJ. Culture, obesity stereotypes, self-esteem, and the "thin ideal": a social identity perspective. J Youth Adolesc. 2004;33:307-17. https://doi.org/10.1023/B: JOYO.0000032639.71472.19.

45. De Zwaan M, Enderle J, Wagner S, et al. Anxiety and depression in bariatric surgery patients: a prospective, follow-up study using structured clinical interviews. J Affect Disord. 2011;133:61-8. https://doi.org/10.1016/j.jad.2011.03.025.

46. Guisado JA, Vaz FJ, Alarcón J, et al. Psychopathological status and interpersonal functioning following weight loss in morbidly obese patients undergoing bariatric surgery. Obes Surg. 2002;12:835-40. https://doi.org/10.1381/096089202320995664.

47. Busetto L, Buongiorno F, Schiff S. Studies on body image changes after bariatric surgery in adults. In: Cuzzolaro M, Fassino S, editors. Body image, eating, weight a guid to assessment, Treat. Prev. Cham: Springer International Publishing; 2018. p. 233-45. https:// doi.org/10.1007/978-3-319-90817-5_17.

48. Munoz D, Chen EY, Fischer S, et al. Changes in desired body shape after bariatric surgery. Eat Disord. 2010;18:347-54. https://doi.org/ 10.1080/10640266.2010.490126.

49. Legenbauer T, Müller A, de Zwaan M, et al. Body image and body avoidance nine years after bariatric surgery and conventional weight loss treatment. Front Psychiatry. 2020;10:1-12. https://doi. org/10.3389/fpsyt.2019.00945.

50. Coulman KD, MacKichan F, Blazeby JM, et al. Patient experiences of outcomes of bariatric surgery: a systematic review and qualitative synthesis. Obes Rev. 2017;18:547-59. https://doi.org/10.1111/obr. 12518.

51. Leonhard ML, Barry NJ. Body image and obesity: effects of gender and weight on perceptual measures of body image. Addict Behav. 1998;23:31-4. https://doi.org/10.1016/S0306-4603(97)00017-8.

52. Muttarak R. Normalization of plus size and the danger of unseen overweight and obesity in England. Obesity. 2018;26:1125-9. https://doi.org/10.1002/oby.22204.

53. Robinson E. Overweight but unseen: a review of the underestimation of weight status and a visual normalization theory. Obes Rev. 2017;18:1200-9. https://doi.org/10.1111/obr.12570.

54. Urdapilleta I, Lahlou S, Demarchi S, et al. Women with obesity are not as curvy as they think: consequences on their everyday life behavior. Front Psychol. 2019;10 https://doi.org/10.3389/fpsyg. 2019.01854.

55. Thaler A, Geuss MN, Mölbert SC, et al. Body size estimation of self and others in females varying in BMI. PLoS One. 2018;13:1-24. https://doi.org/10.1371/journal.pone.0192152.

56. Lacerda RMR, Castanha CR, Castanha AR, et al. Perception of body image by patients undergoing bariatric surgery. Rev Col Bras Cir. 2018;45:1-8. https://doi.org/10.1590/0100-6991e20181793.

Publisher's Note Springer Nature remains neutral with regard to jurisdictional claims in published maps and institutional affiliations. 